\title{
Quaiser et al.
}

\section{Supporting Information}

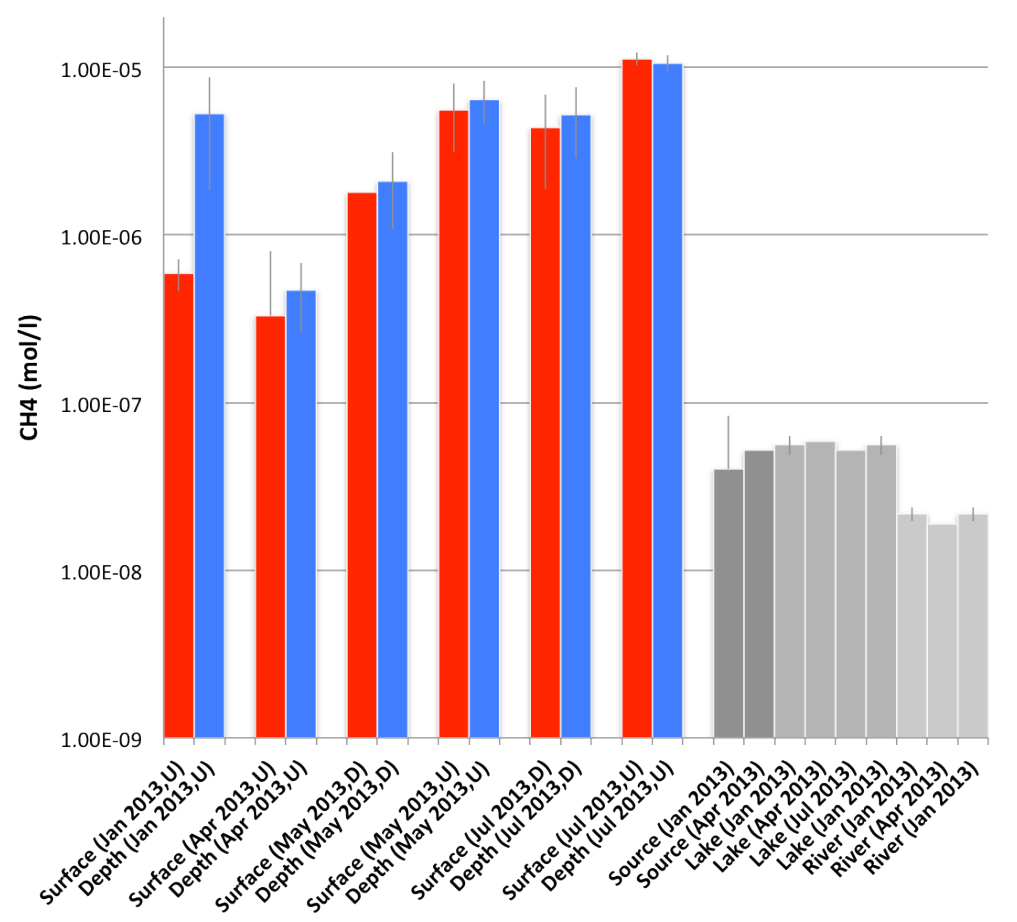

Figure S1. Methane concentrations measured at the sampling sites. Methane concentrations were measured at the two sampling sites (U, upstream; D, downstream) in triplicates distinguishing between depth and surface. For two samples (Surface Jan 2013,D; Surface May 2013,D) only a single measurement was available. Higher methane concentrations were measured in corresponding deep samples with one exception in the samples July 2013, U. Surrounding waters without microbial mat observation are shown in grey: Source, water flow directly upstream of the microbial mats; Lake, lake above the stream without microbial mat; River, main stream with stronger water current. 


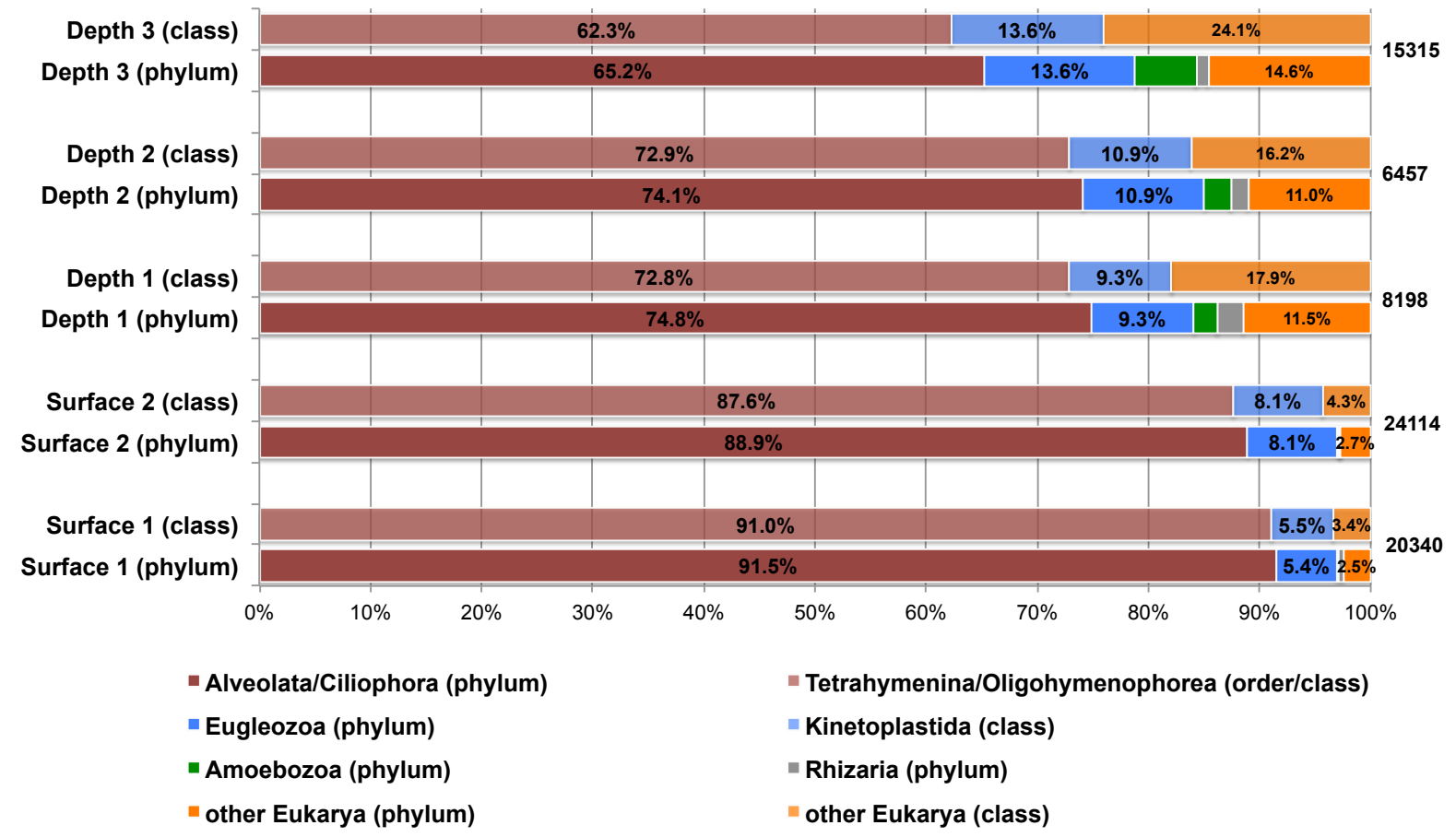

Figure S2. Relative abundance of the eukaryotic members of the microbial community based on the taxonomic affiliation of SSU rRNA read matches at the phylum and class levels. The rRNA reads from each triplicate were combined and normalized average values are shown. The total numbers of eukaryotic matches included in the analysis are indicated. The large majority of eukaryotic matches are affiliated to Tetrahymenina/Oligohymenophorea that contains essentially freshwater grazers such as Tetrahymena species (ranging from $62.3 \%$ to $91.0 \%$ of the eukaryotic matches).
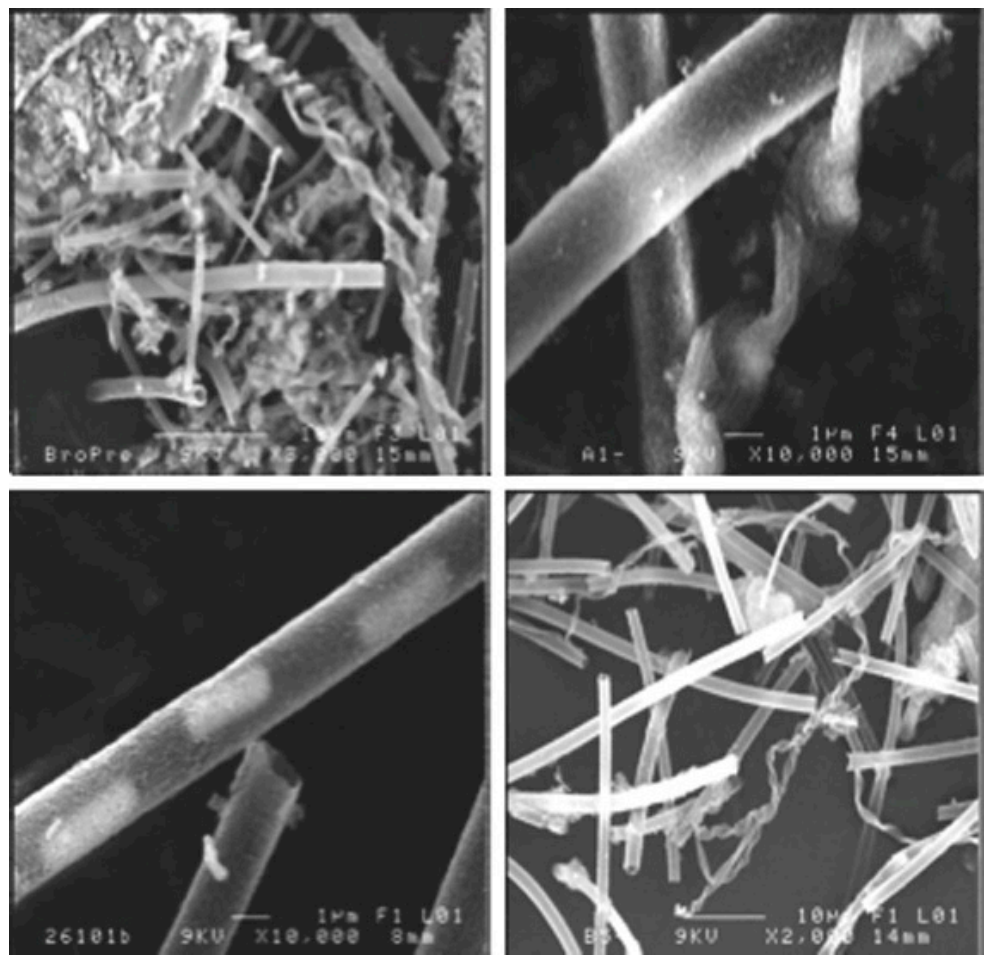

Figure S3. Scanning electron microscopy of the microbial mat showing typical extracellular sheets (Leptothrix) and stalks (Gallionella). 


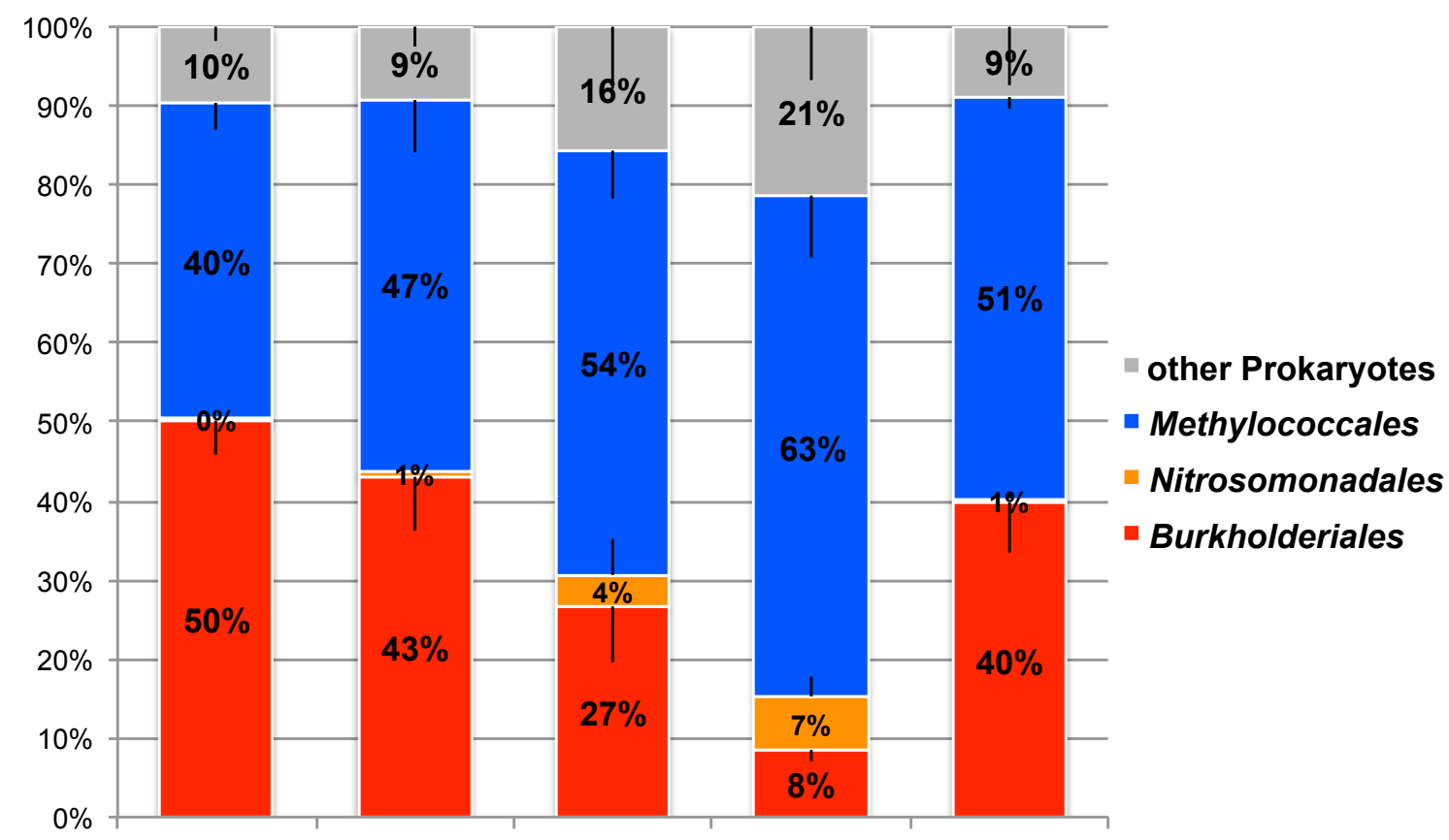

Surface 1 Surface 2 Depth 1 Depth 2 Depth 3

Figure S4. Relative abundance and taxonomic affiliations of OTUs. For illustration purposes confidence intervals from the triplicates are indicated either as negative or positive bars.

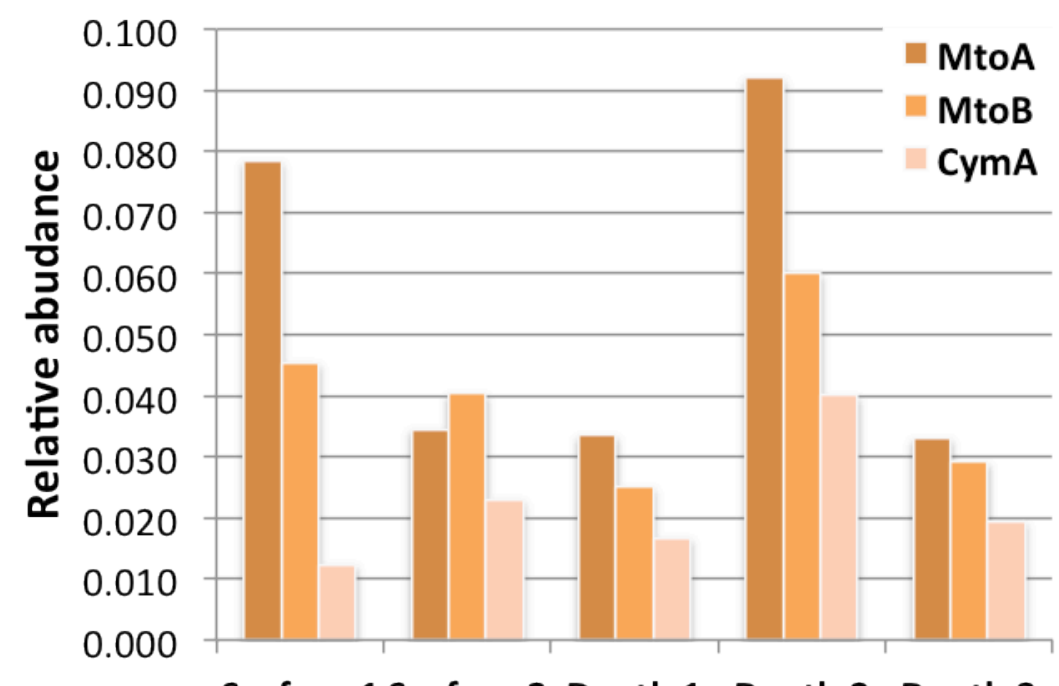

\section{Surface 1 Surface 2 Depth 1 Depth 2 Depth 3}

Figure S5. Relative abundance of reads matching transcripts encoding the three proteins involved in iron oxidation from Sideroxidans lithotrophicus ES-1. MtrA, mtrB, cymA transcripts from each triplicate were combined and normalized average values are shown (in total 158 matches). 
Table S1. General characteristics of the microbial mat samples.

\begin{tabular}{|c|c|c|c|c|c|}
\hline & Surface 1 (S1) & Surface 2 (S2) & Depth 1 (D1) & Depth 2 (D2) & Depth 3 (D3) \\
\hline Sampling date & 08.07 .2010 & 08.07 .2010 & 08.07 .2010 & 08.07 .2010 & 08.07 .2010 \\
\hline Aspect & $\begin{array}{c}\text { Clear orange, } \\
\text { Flocculent }\end{array}$ & $\begin{array}{c}\text { Clear orange, } \\
\text { Flocculent }\end{array}$ & $\begin{array}{l}\text { Brownish-red, } \\
\text { Flocculent with } \\
\text { precipitates }\end{array}$ & $\begin{array}{l}\text { Brownish-red, } \\
\text { Flocculent with } \\
\text { precipitates }\end{array}$ & $\begin{array}{l}\text { Dark brown } \\
\text { Slimy dense } \\
\text { precipitates }\end{array}$ \\
\hline Collection depth & $\begin{array}{c}\text { Surface }(1-3 \mathrm{~cm}) \\
\text { Above D1 }\end{array}$ & $\begin{array}{c}\text { Surface }(1-3 \mathrm{~cm}) \\
\text { Above D2 }\end{array}$ & $\begin{array}{l}\text { Depth }(6-8 \mathrm{~cm}) \\
\text { Below S1 }\end{array}$ & $\begin{array}{l}\text { Depth }(6-8 \mathrm{~cm}) \\
\text { Below S2 }\end{array}$ & Depth (on sed. *) \\
\hline $\mathrm{T}\left({ }^{\circ} \mathrm{C}\right)$ & $16.6^{\circ} \mathrm{C}$ & $15.0^{\circ} \mathrm{C}$ & $15.7^{\circ} \mathrm{C}$ & $15.4^{\circ} \mathrm{C}$ & $15.3^{\circ} \mathrm{C}$ \\
\hline $\mathrm{pH}$ & 6.85 & 6.94 & 6.84 & 6.7 & 6.7 \\
\hline Dissolved oxygen (mg/l) & 1.8 & 1.45 & 2.25 & 1.31 & 1.51 \\
\hline Redoxpotential (mV) & 317 & 306 & 277 & 178 & 197 \\
\hline IC (mg/l) & 2.17 & 1.96 & 1.98 & 1.96 & 1.91 \\
\hline $\mathrm{DOC}(\mathrm{mg} / \mathrm{l})$ & 10.38 & 10.51 & 11.77 & 8.76 & 10.81 \\
\hline $\mathrm{Fe}^{2+}(\mathrm{ppm}$ or $\mathrm{mg} / \mathrm{l})$ & 1.6 & 5.21 & 2.31 & 9.28 & 12.64 \\
\hline $\mathrm{Fe}^{3+}(\mathrm{ppm}$ or $\mathrm{mg} / \mathrm{l})$ & 0.11 & 0.12 & 0.14 & 0.32 & 0.1 \\
\hline Comment & triplicate & triplicate & triplicate & triplicate & Duplicate \\
\hline
\end{tabular}

Table S2. Diversity and richness estimates for the rRNA region analysis.

\begin{tabular}{|c|c|c|c|c|c|}
\hline & Surface 1 (S1) & Surface 2 (S2) & Depth 1 (D1) & Depth 2 (D2) & Depth 3 (D3) \\
\hline \multicolumn{6}{|c|}{ Richness estimation } \\
\hline $\mathrm{N}^{\circ}$ of OTUs & 383 & 244 & 675 & 756 & 2208 \\
\hline Ace & 1211 & 722 & 2443 & 2646 & 4941 \\
\hline Chao & 798.34 & 543.09 & 1677.50 & 1569.40 & 3894.25 \\
\hline \multicolumn{6}{|c|}{ Diversity estimation } \\
\hline Shannon & 2.87 & 2.95 & 4.10 & 4.81 & 4.83 \\
\hline Reciprocal Simpson & 5.34 & 5.99 & 13.02 & 28.07 & 23.79 \\
\hline $\mathrm{N}^{\circ}$ of reads included & 6,392 & 3,254 & 7,089 & 5,749 & 34,116 \\
\hline
\end{tabular}


Table S3. Detailed match counts of non rRNA transcripts to microbial genome sequences

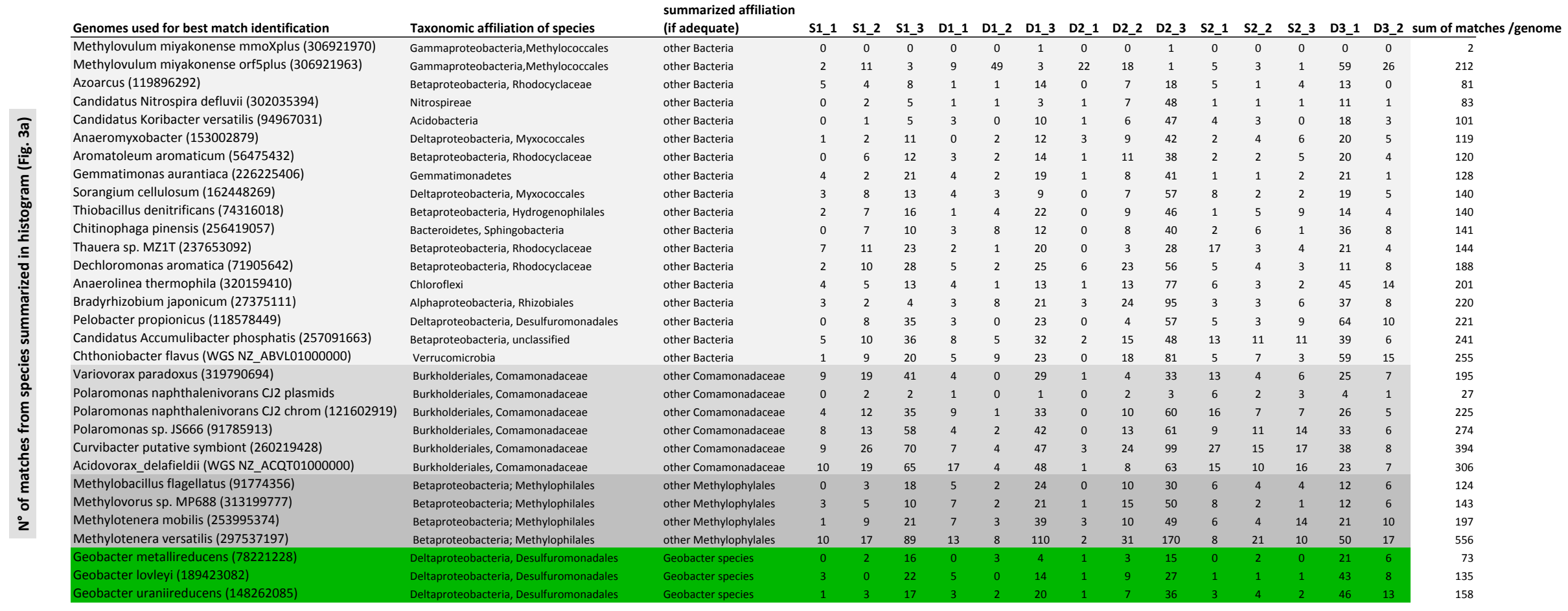

enomes used for best match identification

Taxonomic affiliation of species

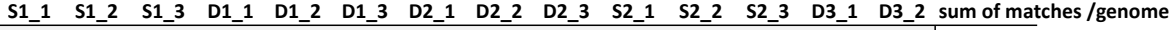
ther Comamondadacea

her Methylophilales

Solibacter usitatus (116222307)

Acidobacteria

(WGS,ABOX00000000)

Acidobacteria
Verrucomicrobia
Vercomicria

\begin{tabular}{llllllllllllll|l}
39 & 105 & 263 & 59 & 98 & 276 & 41 & 190 & 821 & 85 & 62 & 69 & 507 & 122 & 2737 \\
\hline 10 & 91 & 271 & 42 & 11 & 200 & 5 & 61 & 319 & 86 & 49 & 63 & 149 & 34 & 1421
\end{tabular}

hodoferax ferrireducens T118 (89343559)

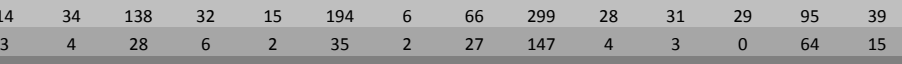

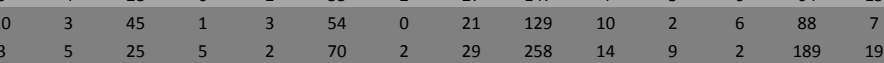

llionella capsiferriformans (302580034)

petoxydans lithotrophicus (2915
pholodnii (170774137)

Methylococcus capsulatus (66270661)

Crenothrix polyspora

Burkholderiale,
Gallionellaceae

Gallionellacea

Methylobacter tundripaludum (344939781) 